\title{
THE RESPONSE OF A TWO-DEGREE-OF-FREEDOM DYNAMIC SLIDING SYSTEM SUBJECTED TO UNI-DIRECTIONAL HORIZONTAL DYNAMIC AND SEISMIC EXCITATIONS
}

\author{
G.C. MANOS, G. KOIDIS \& M. DEMOSTHENOUS \\ Laboratory of Experimental Strength of Materials and Structures, Dept. of Civil Engineering, Aristotle University, \\ Thessaloniki, Greece.
}

\begin{abstract}
An investigation is presented that studies the response of a two-degree-of-freedom dynamic sliding system; that is a single-degree-of-freedom oscillator being fixed on top of a rigid block that can slide on its supporting shaking table along a horizontal axis when subjected to unidirectional dynamic or earthquake excitations along this horizontal axis. This problem appears to be of interest in predicting the dynamic and earthquake response of superstructures supported on a large foundation block capable of horizontal sliding by means of seismic sliding isolators. Special mock-ups are tested at the shaking table of Aristotle University for this purpose utilizing horizontal simulated earthquake excitations based on prototype earthquake ground motion recordings. The numerical results, which were obtained by a computer software developed for this purpose, are compared with the corresponding experimental measurements. The measured acceleration and displacement responses of the mock-ups appear to be, in all the examined cases, in reasonably good agreement with the numerical predictions. However, in certain cases, the numerically predicted sliding displacement of the rigid block appears to have an offset that differs from the sliding response that was observed during the experiments. This is more pronounced when there is no spring linking the rigid block with the shaking table and must be attributed to manufacturing tolerances of the mock-ups. It is demonstrated that the developed software, although it tries to represent such a rather complex problem in a simplified manner, can be useful at the preliminary design stage of structural systems resting on sliding isolators. Results from various experimental and numerical tests demonstrate that the oscillator's response is generally made smaller by the sliding of the rigid block. This is true for a wide range of frequencies; however, there is a relatively narrow frequency window in which the oscillator's response is amplified. This frequency window has a peak value that is slightly higher than the oscillator's eigen-frequency when it is considered to be a single-degree-offreedom system fixed at its base.

Keywords: Earthquake excitation, numerical simulation, seismic isolation, measurements, sliding rigid block.
\end{abstract}

\section{INTRODUCTION}

This paper presents results from an experimental and numerical investigation that studies the response of a two-degree-of-freedom (2-D.O.F.) dynamic sliding system; that is a singledegree-of-freedom (S.D.O.F.) oscillator being fixed on top of a rigid block that can slide on its supporting shaking table along a horizontal axis as shown in Fig. 1. Aslam et al. [1] dealt with the sliding or rocking response of a rigid block (without an S.D.O.F. oscillator) subjected to horizontal and vertical base motions without considering in their analysis the difference between the static and dynamic coefficients of friction, unlike the present study. They complemented their analysis with a parametric study and a valuable experimental verification. Westermo and Udwadia [2] also studied analytically the sliding dynamic response of a rigid block. Shenton et al. [3] dealt with the complex problem of sliding, rocking, and free-flight dynamic response of a rigid block (without an S.D.O.F. oscillator). They considered in their analysis both the static and kinetic coefficients of friction as well as two-component excitation 
(horizontal and vertical). However, apart from the analytical formulation they did not include either a numerical solution or an experimental verification. Virgin et al. [4] studied the rocking and sliding response of a block under horizontal excitation without considering in their analysis the difference between the static and dynamic coefficients of friction, unlike the present study. Demosthenous [5] studied the sliding response of a rigid block considering, as does the present study, the static and dynamic coefficients of friction. Moreover, he has developed a numerical solution for this problem together with an experimental verification, using sinusoidal or simulated earthquake excitations. However, he considered only the rigid block without the S.D.O.F. oscillator shown in Fig. 1. He also considered in his formulation both the horizontal and vertical components of the excitation. The present study was also extended to deal both with the horizontal and vertical components of the excitation. Due to laboratory difficulties, what is presented here is limited at the moment only to the horizontal component of the excitation. Moreover, the present study assumes that no rocking occurs during sliding [6]. The problem shown in Fig. 1 is studied here numerically as well as experimentally utilizing a special mock-up (Section 6) manufactured and tested at the shaking table of Aristotle University. The used horizontal shaking table motions were based on either sinusoidal motions, as done by Mostaghel et al. [7], having a variety of frequencies and amplitudes or based on horizontal earthquake excitations as done by Mostaghel and Tanbakuchi in their study of the same problem [8]. However, these works did not consider in their formulation the difference between the static and dynamic coefficients of friction, unlike the present study. Moreover, they dealt only analytically with the problem of Fig. 1, by employing actual recordings of prototype earthquake ground motions, as done in this paper, without, however, any experimental validation. In the present study, the laboratory simulations of these recordings were measured exactly during testing, and they were used exactly as measured in the subsequent verification of the numerical simulation where the numerical predictions are compared with the measured response. The problem of Fig. 1 is of interest in predicting the dynamic and earthquake response of superstructures on a large foundation block supported on seismic sliding isolators. Krishnamoorthy and Parikh [9] studied the effect of sliding on multi-story frames, considering the constant coefficient of friction. Usually, the superstructure, the foundation, and the sliding isolators are numerically simulated

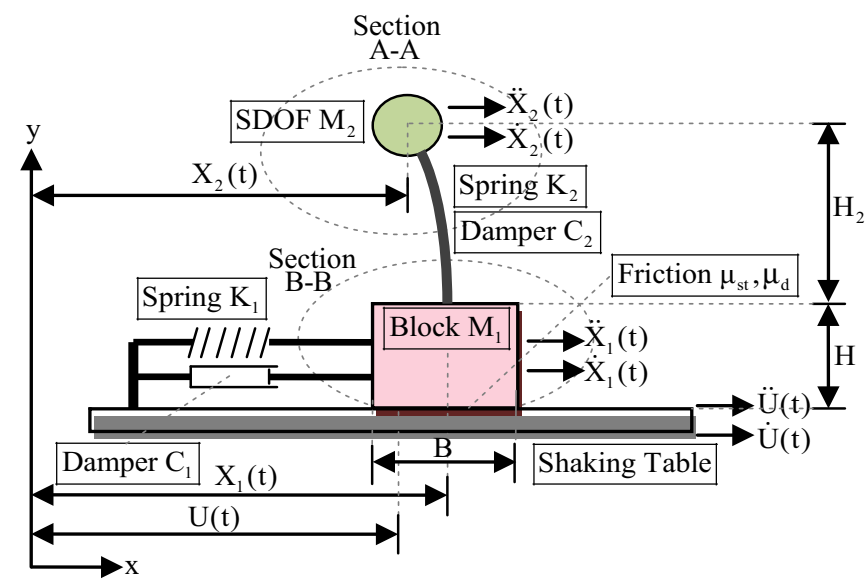

Figure 1: Layout of the problem. 
with complex three-dimensional (3D) models that may result in cumbersome numerical analyses when seeking optimum solutions. The present simplified numerical treatment aimed at facilitating this preliminary design stage. Moreover, concern has been voiced on modeling real sliding conditions. Higashino et al. [10] performed a laboratory and in situ study concerned mainly with the durability of sliding isolators and the variability of friction-sliding characteristics with time. The influence of the actual sliding conditions are taken into account and discussed here by comparing the numerical predictions with laboratory measurements.

\section{PROBLEM LAYOUT}

Figure 1 shows a rectangular rigid block (Block) initially resting on the flat horizontal steel platform of a shaking table (Fig. 1, called Table). A linear single-degree-of-freedom oscillator is fixed on the upper surface of the Block (Fig. 1, called S.D.O.F.). The shaking table moves only in one horizontal direction, thus subjecting the system to uni-directional horizontal excitations. Block movement affects the movement of the S.D.O.F. and vice versa. The contact area between the Block and the shaking table is a horizontal plane where Coulomb friction develops; $\left[\mu_{\mathrm{st}} \cdot\left(\mathrm{M}_{1}+\mathrm{M}_{2}\right) \cdot \mathrm{g}\right.$ is the static friction force and $\mu_{\mathrm{d}} \cdot\left(\mathrm{M}_{1}+\mathrm{M}_{2}\right) \cdot \mathrm{g}$ is the dynamic friction force]. The Block of mass M1 is linked to the shaking table with a horizontal spring of stiffness $\mathrm{K}_{1}$ and a horizontal damper of damping coefficient $\mathrm{C}_{1}$. The S.D.O.F. has stiffness $\mathrm{K}_{2}$ and a coefficient of damping $\mathrm{C}_{2}$. Pure horizontal motion for the S.D.O.F. is assumed. With respect to a fixed coordinate system $\mathrm{x}$ and $\mathrm{y}$, the motion of the shaking table is defined by the displacement $\mathrm{U}(\mathrm{t})$, whereas the corresponding displacement of the Block is $\mathrm{X}_{1}(\mathrm{t})$ and that of the S.D.O.F. is $\mathrm{X}_{2}(\mathrm{t})$. The velocity and acceleration of the shaking table, the Block, and the S.D.O.F. are $\dot{U}(\mathrm{t})$ and $\ddot{U}(\mathrm{t}), \dot{\mathrm{X}}_{1}(\mathrm{t})$ and $\ddot{\mathrm{X}}_{1}(\mathrm{t})$, and $\dot{\mathrm{X}}_{2}(\mathrm{t})$ and $\ddot{\mathrm{X}}_{2}(\mathrm{t})$, respectively. The sliding Block displacement (relative to the shaking table) is equal to $U(t)-X_{1}(t)$. The forces that arise in the system are the following; $F_{\text {ine } 1}$ is the inertial force of the Block, $\mathrm{F}_{\text {ine2 }}$ is the inertial force of the S.D.O.F., $\mathrm{F}_{\mathrm{spr} 1}$ is the force at the Spring 1, $\mathrm{F}_{\mathrm{spr} 2}$ is the force at the Spring 2 (S.D.O.F. restoring force), $\mathrm{F}_{\mathrm{dam} 1}$ is the force at the Damper $1, \mathrm{~F}_{\mathrm{dam} 2}$ is the force at the Damper 2 (S.D.O.F. damping force), and $\mathrm{F}_{\mathrm{fr}}$ is the Coulomb friction force that, when the Block moves with the shaking table, can take a value from zero to $\mu_{\mathrm{st}} \cdot\left(\mathrm{M}_{1}+\mathrm{M}_{2}\right) \cdot \mathrm{g}$, whereas when the Block slides on the shaking table, it takes the value of $\mu_{d} \cdot\left(M_{1}+M_{2}\right) \cdot g$. Generally, positive direction to the right and negative to the left is supposed. Rocking phenomena at the Block-Table interface are not investigated here.

\section{SLIDING DYNAMIC RESPONSE OF THE RIGID BLOCK ALONE}

A simplified version of this problem is shown in Fig. 2. where no S.D.O.F. is placed on top of the Block. All the parameters with the relevant symbols given in Section 2 are also valid here. The forces that arise in this rigid block - shaking table dynamic system [11] are shown in Fig. 2.

A special mock-up has been constructed that represents physically the previously stated problem (Fig. 2). It consisted of a rectangular steel block with dimensions $430 \mathrm{~mm} \times 430 \mathrm{~mm}$ in plan and a height either $205 \mathrm{~mm}$ or $410 \mathrm{~mm}$ (upper part of Fig. 2). This Block was simply resting on the steel platform of the shaking table (lower part of Fig. 2) and was restrained in all other directions so that it could move only in the longitudinal horizontal direction. The mass of this sliding Block was either $124 \mathrm{~kg}$ or $248 \mathrm{~kg}$. The various important physical parameters for this Block sliding response are measured [12], e.g. the coefficients of friction at the contact surface between the Block and the shaking table and the stiffness of the elastic spring. A special series of tests were performed in order to determine the coefficient of 


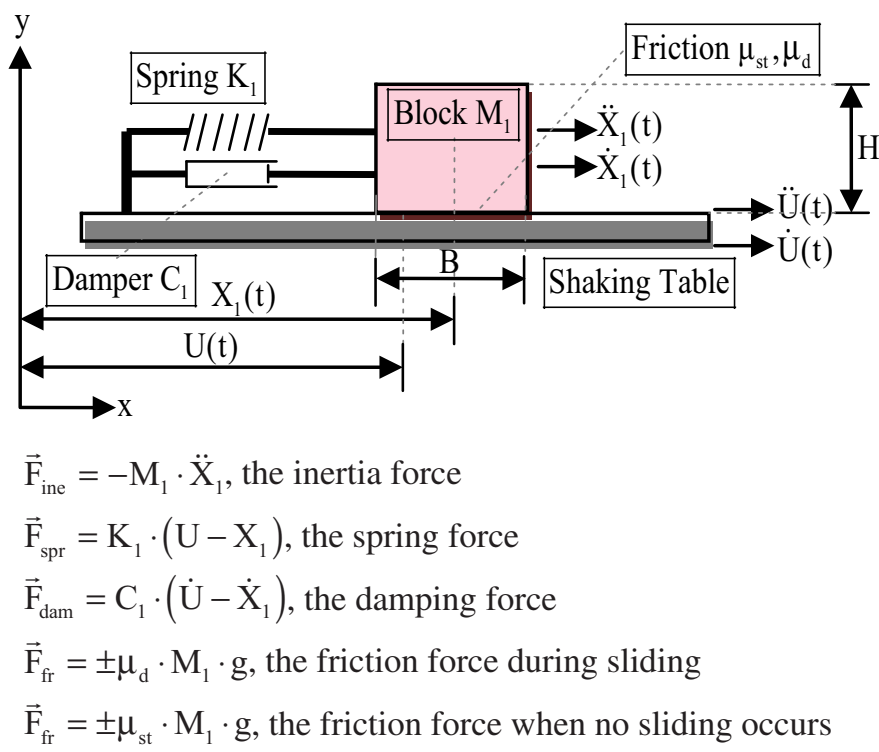

Figure 2: Layout of the mock-up of the sliding Block alone without the S.D.O.F. on top.

friction of the contact surface between the sliding Block and the shaking table. This surface was machined in a special way so that full contact conditions could be ensured between the sliding Block and the steel platform of the shaking table. Moreover, this contact surface was lubricated throughout all experimental sequences [12]. The frequency of the cyclic displacements during these tests varied from $0.1 \mathrm{~Hz}$ to $1.0 \mathrm{~Hz}$. The average values for the coefficient of friction found from these tests are listed below; they were found not to depend on the frequency of the excitation. A total of 10 tests were performed. When the Block's mass was equal to $124 \mathrm{~kg}$, the values of the friction coefficient were: $\mu_{\mathrm{st}}=0.165, \mu_{\mathrm{d}}=0.153$. When the Block's mass was equal to $284 \mathrm{~kg}$, the values of the friction coefficient were: $\mu_{\mathrm{st}}=0.158$, $\mu_{\mathrm{d}}=0.136$.

Another series of tests were conducted in order to accurately measure the stiffness $\mathrm{K}_{1}$ of the spring that was employed to link the Block to the shaking table. As was done for measuring the coefficient of friction, during the tests for measuring the spring stiffness, the frequency of the cyclic displacement was varied from $0.1 \mathrm{~Hz}$ to $1.0 \mathrm{~Hz}$. The stiffness of the certified spring with no pre-stress had an average value $\mathrm{K}_{1}=128.6 \mathrm{kN} / \mathrm{m}$. The stiffness of the certified spring with pre-stress had an average value $\mathrm{K}_{1}=143.0 \mathrm{kN} / \mathrm{m}$. The stiffness of the noncertified spring with pre-stress had an average value $\mathrm{K}_{1}=19.4 \mathrm{kN} / \mathrm{m}$. There was no viscous damper used in this mock-up.

\subsection{Governing relationships and numerical solution}

The following relationships, (1) to (4), govern the sliding of the Block that is linked with a spring $\left(\mathrm{K}_{1}\right)$ and a damper $\left(\mathrm{C}_{1}\right)$ to the shaking table when it is subjected to horizontal acceleration. Starting from an initial condition of no motion, in order for the Block to start sliding, the shaking table acceleration must exceed the value given by the following inequality (1) 


$$
|\ddot{\mathrm{U}}(\mathrm{t})|>\mu_{\mathrm{st}} \cdot \mathrm{g}
$$

In this case, the Block acceleration will be given by eqn (2):

$$
\ddot{X}_{1}(t)=\mu_{d} \cdot g \cdot\left[\operatorname{sgn}\left(\dot{U}(t)-\dot{X}_{1}(t)\right)\right]+\frac{K_{1}}{M_{1}} \cdot\left(U(t)-X_{1}(t)\right)+\frac{C_{1}}{M_{1}} \cdot\left(\dot{U}(t)-\dot{X}_{1}(t)\right)
$$

When the Block is sliding, it is continuously checked for reattachment to the shaking table by examining the absolute value of its velocity relative to the shaking table. If the value of the velocity of the Block relative to the shaking table becomes equal to zero and inequality (3) holds, the Block will keep sliding.

$$
\text { If }\left|\dot{U}(t)-\dot{X}_{1}(t)\right|=0 \text { and } \quad\left|\ddot{U}(t)-\frac{K_{1}}{M_{1}} \cdot\left(U(t)-X_{1}(t)\right)\right|>\mu_{s t} \cdot g
$$

If the value of the velocity of the Block relative to the shaking table becomes equal to zero and inequality (4) holds, the Block is reattached to the shaking table.

$$
\text { If }\left|\dot{U}(t)-\dot{X}_{1}(t)\right|=0 \text { and }\left|\ddot{U}(t)-\frac{K_{1}}{M_{1}} \cdot\left(U(t)-X_{1}(t)\right)\right| \leq \mu_{s t} \cdot g
$$

The above conditions are included in computer software that utilizes a time step-by-step integration process incorporating an iteration scheme to reach acceptable limits of convergence. In this step-by-step integration process, eqns (5) and (6) are used for defining the velocity and displacement of the Block at time step $t_{i}$.

$$
\begin{aligned}
& \dot{\mathrm{X}}\left(\mathrm{t}_{\mathrm{i}}\right)=\dot{\mathrm{X}}\left(\mathrm{t}_{\mathrm{i}-1}\right)+\left(\ddot{\mathrm{X}}\left(\mathrm{t}_{\mathrm{i}-1}\right)+\ddot{\mathrm{X}}\left(\mathrm{t}_{\mathrm{i}}\right)\right) \cdot \frac{\Delta \mathrm{t}}{2} \\
& \mathrm{X}\left(\mathrm{t}_{\mathrm{i}}\right)=\mathrm{X}\left(\mathrm{t}_{\mathrm{i}-1}\right)+\left(\dot{\mathrm{X}}\left(\mathrm{t}_{\mathrm{i}-1}\right)+\dot{\mathrm{X}}\left(\mathrm{t}_{\mathrm{i}}\right)\right) \cdot \frac{\Delta \mathrm{t}}{2}
\end{aligned}
$$

This computer software was next validated (Section 3.2) by numerically simulating the dynamic sliding response of the tested Block and by comparing the response measured in the laboratory with the corresponding numerical predictions. In this numerical simulation, all the important physical parameters of the problem at hand, such as the friction coefficients, spring stiffness, Block mass, and the shaking table motion, are the input to this computer software with values found from the laboratory measurements. Thus, the measured sliding response of the mock-up could be directly compared to numerical predictions made by this developed computer software.

\subsection{Comparison between experimental measurements and numerical predictions}

\subsubsection{Test employing sinusoidal excitation $3.5 \mathrm{~Hz}$ with certified spring}

The comparison between predicted and measured Block acceleration and sliding displacement response is presented in Fig. 3a and b, respectively. As can be seen in these figures, good agreement is obtained between predicted and measured response. 

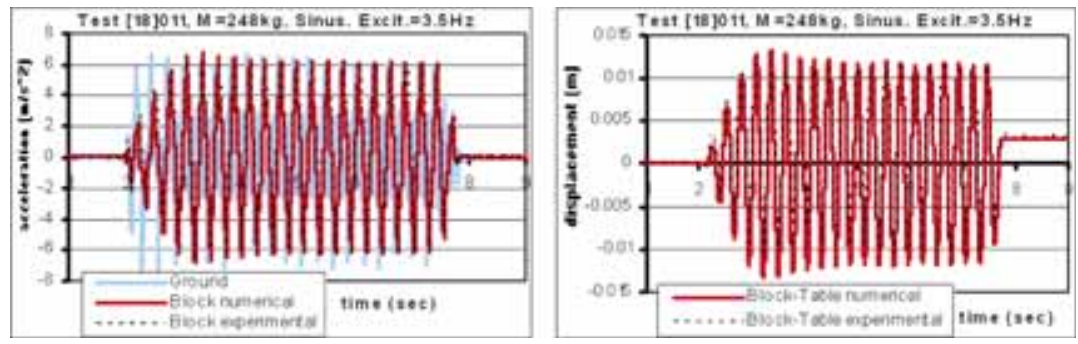

Figure 3: (a) Table (Ground) and Block (observed and predicted) acceleration response. (b) Sliding displacement of the Block relative to the shaking table.
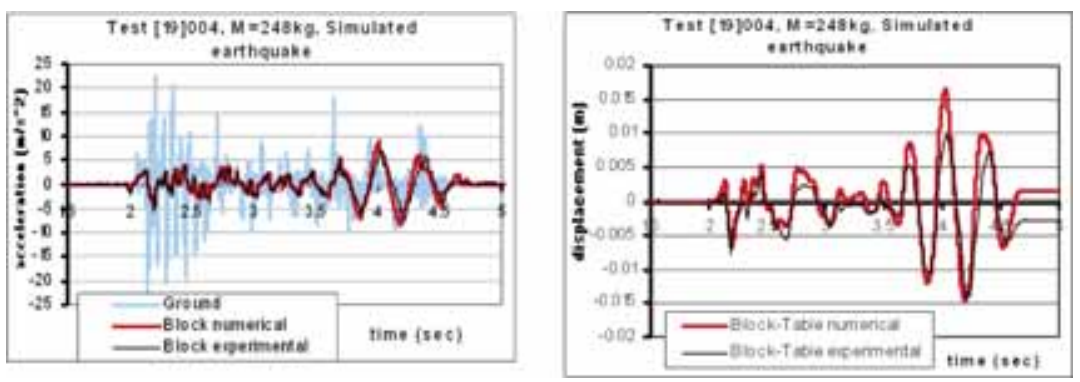

Figure 4: (a) Table (Ground) and Block (observed and predicted) acceleration response.

(b) Sliding displacement of the Block relative to the shaking table.

\subsubsection{Test employing a simulated earthquake excitation with certified spring}

The comparison between predicted and measured Block acceleration and sliding displacement response is presented in Fig. $4 \mathrm{a}$ and $\mathrm{b}$. The Block is linked to the shaking table with the certified spring (with pre-stress). This time the excitation is a simulated earthquake motion, based on the Kern Country 1953 prototype earthquake horizontal acceleration recording. As can be seen, good agreement is obtained between predicted and measured Block acceleration response (Fig. 4a). One of the important design parameters in base isolation schemes utilizing sliders is the sliding displacement. Choia and Tung [13] performed a parametric study on the sliding displacement using many earthquake records. The sliding displacement response will be particularly studied and discussed in this paper. The predicted Block sliding displacement response correlates reasonably well with the one measured in the laboratory in the time domain. Moreover, the predicted sliding displacement response, in terms of amplitude, is in reasonable agreement with the one measured in the laboratory (Fig. 4b).

\subsubsection{Test employing sinusoidal excitation $1.5 \mathrm{~Hz}$ without spring}

The comparison between predicted and measured Block acceleration and sliding displacement response is again presented in Fig. 5a and b. The Block this time is resting on the shaking table without any spring link attachment. The excitation of the shaking table is sinusoidal at $1.5 \mathrm{~Hz}$. As can be seen, good agreement is obtained between predicted and measured Block acceleration response. The predicted Block sliding displacement response correlates reasonably well with the one measured in the laboratory in the time domain. However, the 

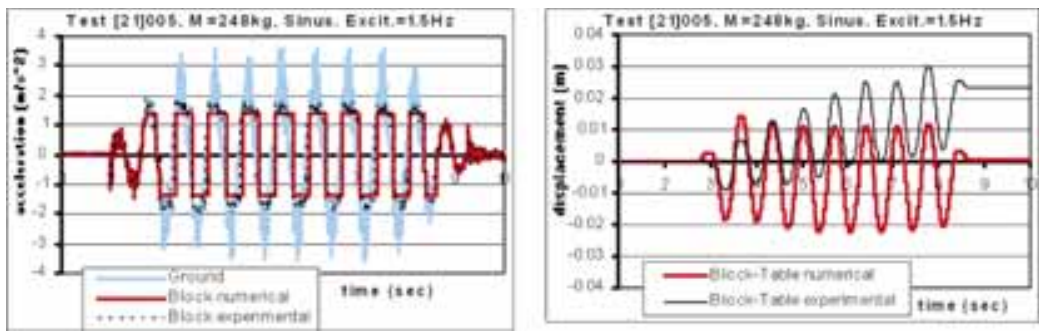

Figure 5: (a) Table (Ground) and Block (observed and predicted) acceleration response.

(b) Sliding displacement of the Block relative to the shaking table.
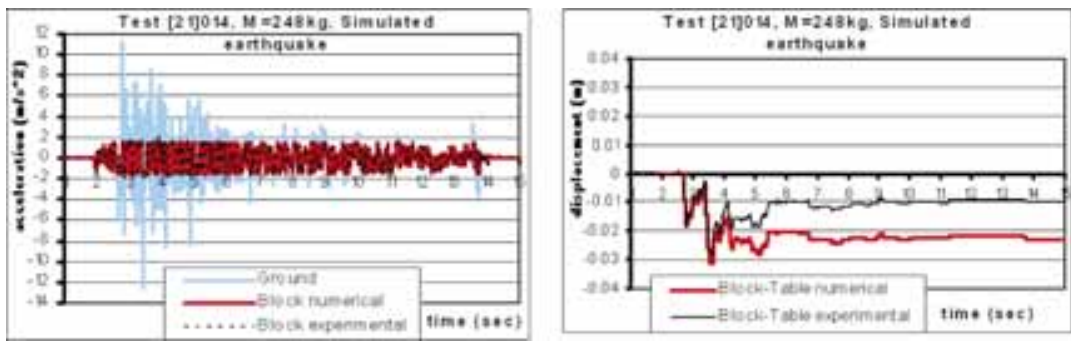

Figure 6: (a) Table (Ground) and Block (observed and predicted) acceleration response. (b) Sliding displacement of the Block relative to the shaking table.

predicted sliding displacement response, in terms of amplitude, is significantly larger than the one measured in the laboratory. It is interesting to note that the cumulative sliding displacement, which appears as an offset from the initial zero displacement condition in the measurements, is not predicted by the software. This measured cumulative (offset) sliding displacement response may be attributed to manufacturing tolerances, whereby the contact surface between the Block and the shaking table deviates from the ideal horizontal plane.

\subsubsection{Test employing simulated earthquake excitation without spring}

The comparison between predicted and measured Block acceleration response is presented in Fig. 6a, whereas the predicted Block sliding response is presented in Fig. 6b. The Block is resting on the shaking table without employing any spring attachment; however, this time a simulated earthquake excitation is used. As can be seen, good agreement is obtained between the predicted and measured Block acceleration response. The predicted Block sliding displacement response correlates reasonably well with the one measured in the laboratory in the time domain as well as in terms of maximum amplitude. It is again interesting to note that there is a cumulative sliding displacement that appears as an offset from the initial zero displacement condition in the predicted response that is much larger than the one observed during the experiment. This deviation between predictions and measured sliding displacement response may be again attributed, as was done before, to manufacturing tolerances, whereby the contact surface between the Block and the shaking table deviates from the ideal horizontal plane. This may result in somewhat larger maximum friction force in one direction than the maximum friction force in the opposite direction. 
The numerical predictions presented in this section confirm all behavioral observations of the studied Block sliding response, as recorded during this extensive experimental sequence. Deviations between predicted and measured maximum and cumulative sliding displacement response are considerably reduced when a spring connects the rigid block with the shaking table. Inverted pendulum sliding isolators, apart from sliding, possess such a spring balancing force property [14-16]. Based on these observations, the investigation is next extended to deal with the more complex dynamic system described in Section 2; that is the 2-D.O.F. system (Fig. 1), where an S.D.O.F. is fixed on top of the sliding block.

\section{GOVERNING RELATIONSHIPS OF THE 2-D.O.F. SYSTEM}

The governing relationships of the 2-D.O.F. sliding system described in Section 1 are stated here. These are an extension of the relationships given in Section 3.1 for the sliding Block alone.

a. When the Block is initially attached to the Table, it is $\mathrm{X}_{1}=\mathrm{U}, \dot{\mathrm{X}}_{1}=\dot{\mathrm{U}}, \ddot{\mathrm{X}}_{1}=\ddot{\mathrm{U}}$. Sliding will start when the inequality (7) becomes valid:

$$
\left|\overrightarrow{\mathrm{F}}_{\mathrm{spr} 1}+\overrightarrow{\mathrm{F}}_{\mathrm{dam} 1}+\overrightarrow{\mathrm{F}}_{\text {ine } 1}+\overrightarrow{\mathrm{F}}_{\text {ine } 2}\right|>\overrightarrow{\mathrm{F}}_{\mathrm{fr}} \Rightarrow
$$

The dynamic equilibrium of the S.D.O.F alone (Section A-A, Fig. 1) results in the following:

$$
\left|\mathrm{M}_{1} \cdot \ddot{\mathrm{X}}_{1}+\mathrm{K}_{2} \cdot\left(\mathrm{X}_{1}-\mathrm{X}_{2}\right)+\mathrm{C}_{2} \cdot\left(\dot{\mathrm{X}}_{1}-\dot{\mathrm{X}}_{2}\right)\right|>\mu_{\mathrm{st}} \cdot\left(\mathrm{M}_{1}+\mathrm{M}_{2}\right) \cdot \mathrm{g}
$$

b. When the Block is sliding from this initial condition, eqns (9) and (10) are valid:

$$
\begin{gathered}
\Sigma \overrightarrow{\mathrm{F}}_{\mathrm{x}}=0 \Rightarrow \overrightarrow{\mathrm{F}}_{\mathrm{spr} 2}+\overrightarrow{\mathrm{F}}_{\mathrm{dam} 2}+\overrightarrow{\mathrm{F}}_{\mathrm{ine} 2}=0 \Rightarrow(\text { Section A-A, Fig. 1) } \\
\ddot{\mathrm{X}}_{2}=\frac{\mathrm{K}_{2}}{\mathrm{M}_{2}} \cdot\left(\mathrm{X}_{1}-\mathrm{X}_{2}\right)+\frac{\mathrm{C}_{2}}{\mathrm{M}_{2}} \cdot\left(\dot{\mathrm{X}}_{1}-\dot{\mathrm{X}}_{2}\right) \\
\Sigma \overrightarrow{\mathrm{F}}_{\mathrm{x}}=0 \Rightarrow \overrightarrow{\mathrm{F}}_{\mathrm{spr} 1}+\overrightarrow{\mathrm{F}}_{\mathrm{dam} 1}+\overrightarrow{\mathrm{F}}_{\text {inel }}+\overrightarrow{\mathrm{F}}_{\mathrm{ine} 2}+\overrightarrow{\mathrm{F}}_{\mathrm{fr}}=0 \Rightarrow(\text { Section B-B, Fig. } 1): \\
\ddot{\mathrm{X}}_{1}=\frac{\mathrm{K}_{1}}{\mathrm{M}_{1}} \cdot\left(\mathrm{U}-\mathrm{X}_{1}\right)+\frac{\mathrm{C}_{1}}{\mathrm{M}_{1}} \cdot\left(\dot{\mathrm{U}}-\dot{\mathrm{X}}_{1}\right)-\frac{\mathrm{M}_{2}}{\mathrm{M}_{1}} \cdot \ddot{\mathrm{X}}_{2}+\mu_{\mathrm{d}} \cdot \frac{\mathrm{M}_{1}+\mathrm{M}_{2}}{\mathrm{M}_{1}} \cdot \mathrm{g} \cdot \operatorname{sgn}\left(\dot{\mathrm{U}}-\dot{\mathrm{X}}_{1}\right)
\end{gathered}
$$

From eqns (9) and (10), acceleration of the Block is given by eqn (11):

$$
\begin{gathered}
\ddot{X}_{1}=\frac{K_{1}}{M_{1}} \cdot\left(U-X_{1}\right)+\frac{C_{1}}{M_{1}} \cdot\left(\dot{U}-\dot{X}_{1}\right)-\frac{M_{2}}{M_{1}} \cdot\left[\frac{K_{2}}{M_{2}} \cdot\left(X_{1}-X_{2}\right)+\frac{C_{2}}{M_{2}} \cdot\left(\dot{X}_{1}-\dot{X}_{2}\right)\right], \\
+\mu_{d} \cdot \frac{M_{1}+M_{2}}{M_{1}} \cdot g \cdot \operatorname{sgn}\left(\dot{U}-\dot{X}_{1}\right)
\end{gathered}
$$

c. When the Block is reattached to the Table it is $\dot{X}_{1}=\dot{U}, \ddot{X}_{1}=\ddot{U}$. In this case, the acceleration of the S.D.O.F. is given by eqn (12). It must be noted that the Block has been 
moved on the Table to a position that will be, in general, different from the initial position of step (a). Thus, the force at spring 1 will have a non-zero value.

$$
\begin{gathered}
\Sigma \overrightarrow{\mathrm{F}}_{\mathrm{x}}=0 \Rightarrow \overrightarrow{\mathrm{F}}_{\mathrm{spr} 2}+\overrightarrow{\mathrm{F}}_{\mathrm{dam} 2}+\overrightarrow{\mathrm{F}}_{\mathrm{ine} 2}=0 \Rightarrow(\text { Section A-A, Fig. 1) } \\
\ddot{\mathrm{X}}_{2}=\frac{\mathrm{K}_{2}}{\mathrm{M}_{2}} \cdot\left(\mathrm{X}_{1}-\mathrm{X}_{2}\right)+\frac{\mathrm{C}_{2}}{\mathrm{M}_{2}} \cdot\left(\dot{\mathrm{X}}_{1}-\dot{\mathrm{X}}_{2}\right)
\end{gathered}
$$

d While the Block is reattached to the Table, it is $\dot{X}_{1}=\dot{U}, \ddot{X}_{1}=\ddot{U}$. Sliding will start again when the inequality (13) becomes valid:

$$
\left|\overrightarrow{\mathrm{F}}_{\text {sprl }}+\overrightarrow{\mathrm{F}}_{\text {daml }}+\overrightarrow{\mathrm{F}}_{\text {inel }}+\overrightarrow{\mathrm{F}}_{\text {ine2 }}\right|>\overrightarrow{\mathrm{F}}_{\text {fr }} \Rightarrow
$$

The dynamic equilibrium of the S.D.O.F alone (Section A-A, Fig. 1) results in the following:

$$
\left|\mathrm{K}_{1} \cdot\left(\mathrm{U}-\mathrm{X}_{1}\right)-\mathrm{M}_{1} \cdot \ddot{\mathrm{X}}_{1}-\mathrm{K}_{2} \cdot\left(\mathrm{X}_{1}-\mathrm{X}_{2}\right)-\mathrm{C}_{2} \cdot\left(\dot{\mathrm{X}}_{1}-\dot{\mathrm{X}}_{2}\right)\right|>\mu_{\mathrm{st}} \cdot\left(\mathrm{M}_{1}+\mathrm{M}_{2}\right) \cdot \mathrm{g}
$$

The above governing equations are utilized in the numerical investigation that is presented in Section 5. These equations will take a somewhat simpler form when there is no spring 1 and damper 1 than the more general case, which was considered here and corresponds to the general layout of the problem depicted in Fig. 1. This relatively simple layout without spring 1 and damper 1, representing a simplification of the general layout shown in Fig. 1, was utilized in the experimental investigation to be discussed next in Section 6.

\section{NUMERICAL INVESTIGATION OF THE 2-D.O.F. SYSTEM}

The relationships given in Section 4 are the governing differential equations as well as the inequalities that define the sliding slip-stick behavior of the 2-D.O.F. dynamic system under consideration, when it is subjected to unidirectional horizontal $\ddot{U}(\mathrm{t})$ acceleration at its base. These governing relationships represent the most general case for the sliding behavior of such a 2-D.O.F. system $[2,3,7-9,12]$. These governing relationships are included in a computer software with the logical diagram shown in Fig. 7. The solution is reached utilizing a step-by-step time integration process, which is also part of this software.

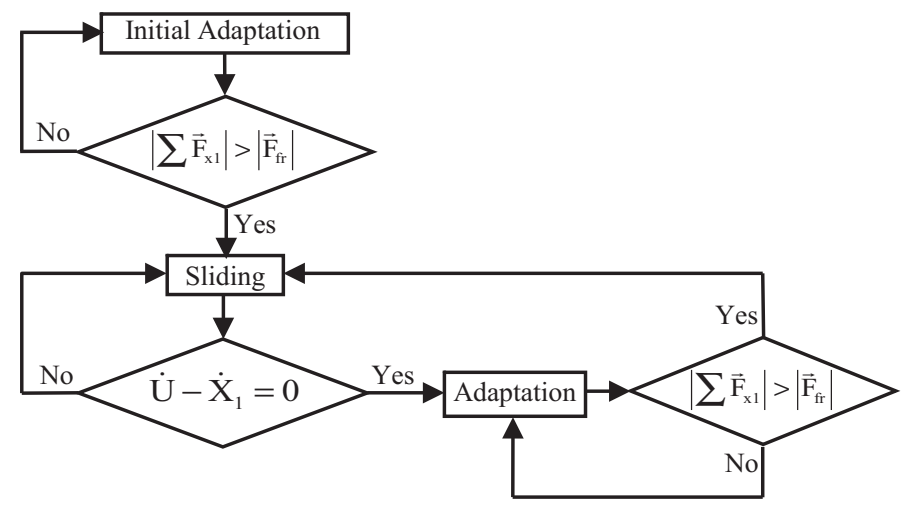

Figure 7: Flow chart of the numerical solution. 
Starting from an initial condition of no motion, the solution proceeds with this step-by-step time integration process checking through the governing relationships at each time step (i-1). This is done by considering all known parameters of the problem at this step, in order to predict the condition, either slip or stick, of the next step (i) as well as all response parameters at this step.

The following simple numerical integration eqns (15) and (16) are utilized for calculating the velocity and displacement at a time step $t_{1}$ :

$$
\begin{aligned}
& \dot{X}\left(t_{i}\right)=\dot{X}\left(t_{i-1}\right)+\left(\ddot{X}\left(t_{i-1}\right)+\ddot{X}\left(t_{i}\right)\right) \cdot \frac{\Delta t}{2} \\
& X\left(t_{i}\right)=X\left(t_{i-1}\right)+\left(\dot{X}\left(t_{i-1}\right)+\dot{X}\left(t_{i}\right)\right) \cdot \frac{\Delta t}{2}
\end{aligned}
$$

At each step (i-1) a number of (j) iterations are performed in order to predict the acceleration of the next time step (i) for either block $\ddot{X}_{1}(i)$ or the S.D.O.F. $\ddot{X}_{2}(i)$. These target accelerations are found by introducing a parameter $\delta$, which is set to a relatively very small value (e.g. $\delta=0.00001 \mathrm{~m} / \mathrm{s}^{2}$ ). The following inequalities (17) and (18) are then utilized as a convergence criterion included in the software for a given set of consecutive iterative solutions $\mathrm{j}-1$ and $\mathrm{j}$ :

$$
\begin{aligned}
& \left|\ddot{X}_{1}{ }^{j}(i)-\ddot{X}_{1}{ }^{j-1}(i)\right| \leq \delta \\
& \left|\ddot{X}_{2}{ }^{j}(i)-\ddot{X}_{2}{ }^{j-1}(i)\right| \leq \delta
\end{aligned}
$$

Ideally, both these inequalities (17) and (18) must be valid in order to satisfy the convergence of this process and stop at iteration (j) adopting the acceleration values block $\ddot{X}_{1}{ }^{j}(\mathrm{i})$ or S.D.O.F. $\ddot{X}_{2}{ }^{j}$ (i) as the final values for time step (i). Normally, about six iterations are sufficient to predict the acceleration at a time step (i) with such accuracy $(\delta)$. This convergence criterion is usually satisfied when the value for $\delta=0.00001 \mathrm{~m} / \mathrm{s}^{2}$ is larger than the round-off accuracy of the numbers inherent in the numerical solution. An instability in this convergence process is observed only when the Block slides during time step (i-1) and the sign of the relative velocity between the Block and the shaking table predicted at iteration step (j) for time step (i), $\operatorname{sgn}\left(\dot{U}^{\mathrm{j}}(\mathrm{i})-\dot{\mathrm{X}}_{1}^{\mathrm{j}}(\mathrm{i})\right)$, is the opposite of the corresponding sign of this relative velocity predicted for time step (i) at iteration step $(\mathrm{j}-1), \operatorname{sgn}\left(\dot{\mathrm{U}}^{\mathrm{j}-1}(\mathrm{i})-\dot{\mathrm{X}}_{1}^{\mathrm{j}-1}(\mathrm{i})\right)$. This instability in the convergence of the numerical solution is dealt with by performing for time step (i) at every iteration step (j) a check of the governing equations that will prove that there is either a stick condition or a sliding condition.

\section{EXPERIMENTAL INVESTIGATION OF THE 2-D.O.F. SYSTEM}

A special mock-up has been constructed (Fig. 8a), which represents the dynamic system depicted in Fig. 1. This mock-up consists of a steel block, with dimensions $0.33 \mathrm{~m} \times 0.53 \mathrm{~m}$ $\times 0.53 \mathrm{~m}$ and mass equal to $325 \mathrm{~kg}$. At the upper side of this Block, a vertical rod is fixed that supports on its top a steel mass varying during the test sequence having values equal to $45 \mathrm{~kg}$, $90 \mathrm{~kg}$, or $201 \mathrm{~kg}$. The steel rod diameter was equal to $14 \mathrm{~mm}$ or $20 \mathrm{~mm}$. The mass of the steel rod is negligible compared to the steel mass being supported at its top. This steel massvertical steel rod cantilever represents an S.D.O.F. oscillator (Fig. 8a and b). The vertical 

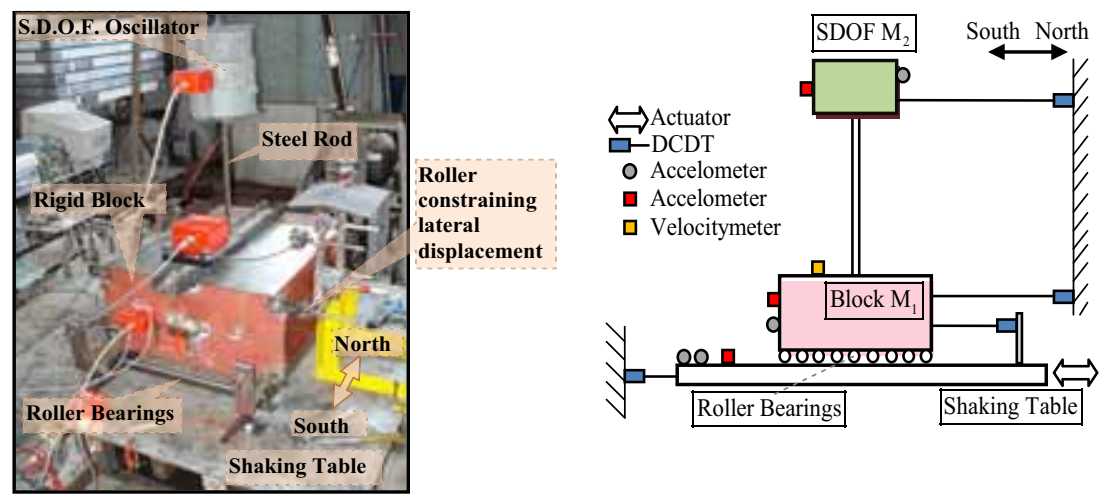

Figure 8: (a) Laboratory mock-up. (b) Instrumentation for measuring the excitation as well as the response of the mock-up.

distance from the center of the mass of the S.D.O.F. up to the upper side of the block, e.g. the plane where the S.D.O.F. is presumed fixed, was set equal to $0.44 \mathrm{~m}$ or $0.76 \mathrm{~m}$. Through the variation of the mass of the oscillator, and of the diameter and/or height of the steel rod, the eigen-frequency of this S.D.O.F. could take values equal to $1.125 \mathrm{~Hz}, 1.625 \mathrm{~Hz}, 1.75 \mathrm{~Hz}$, $2.15 \mathrm{~Hz}$, or $2.65 \mathrm{~Hz}$. The whole dynamic system, that is the S.D.O.F. and the supporting Block, was simply resting on the horizontal plane of the shaking table steel platform utilizing a roller-bearing device placed at the contact surface between the bottom side of the Block and the upper side of the shaking table (Figs 1 and $8 b$ ).

As shown in Fig. 8b, the shaking table can move horizontally only in the North-South direction. The Block, through the roller-bearing support could also slide in this longitudinal (North-South) horizontal direction, whereas it was constrained against movement in the transverse horizontal direction (East-West) by special lateral displacement roller ties employed for this purpose. Two distinct types of horizontal motions were introduced to excite the studied dynamic system. The first type of shaking table motion was a horizontal sinusoidal excitation with a frequency varying during tests from $1 \mathrm{~Hz}$ to $3.4 \mathrm{~Hz}$ and with amplitudes varying during tests from $0.1 \mathrm{~m} / \mathrm{s}^{2}$ to $5 \mathrm{~m} / \mathrm{s}^{2}$. The frequency and amplitude of this horizontal sinusoidal excitation was kept constant during each test. The second type of shaking table motion was a horizontal excitation that was a laboratory simulation based on the actual horizontal recording of one of the horizontal components of the prototype Kern County California 1953 earthquake. All the physical quantities entering into the problem, such as the mass of the Block, the mass of S.D.O.F., the coefficients of friction of the roller-bearing device, the damping coefficient of the S.D.O.F. were measured before the experimental sequence commenced. The results reported here were obtained with a mock-up having neither a spring nor a viscous damper connection between the Block and the shaking table (Figs 1 and $8 \mathrm{~b}$ ). Care was taken during the test sequence to prohibit any rocking and uplift of the Block from the supporting roller bearing. A total of 150 sliding tests were performed. In order to define the coefficients of friction of the roller-bearing device, special sliding tests were performed when mass $M_{1}$ of the block was equal to $325 \mathrm{~kg}$. The value for static coefficient of friction was found equal to 0.020 and that for the dynamic coefficient of friction equal to 0.012 . The equivalent viscous damping ratio of the S.D.O.F. had a relatively small value of the order of $1 \%$ of critical damping. A number of 
displacement, velocity, and acceleration sensors were provided in order to accurately record the characteristics of the exciting shaking table motion as well as the response of both the Block at the center of its mass, its upper side, as well as at the center of the mass of the S.D.O.F. (Fig. 8a and b). A set of displacement transducers recorded the displacement of the shaking table, the Block, and the S.D.O.F. with respect to a motionless coordinate system. In addition, the sliding displacement of the Block (relative to the shaking table) was also measured directly by an additional sensor (Fig. 8b). An automatic data acquisition system with a sampling rate sufficient for accurately measuring the excitation and the response was utilized during testing.

\section{COMPARISON BETWEEN EXPERIMENTAL MEASUREMENTS AND NUMERICAL PREDICTIONS FOR THE 2-D.O.F. SYSTEM}

The computer software presented in Section 5 was next validated by numerically simulating the dynamic sliding response of the 2-D.O.F. system of Section 6 and by comparing the response measured in the laboratory with the corresponding numerical predictions. Selective numerical results are presented here and compared with the corresponding experimental measurements. Subsection (7.1) includes results obtained from tests employing sinusoidal excitation, whereas Subsection (7.2) includes results obtained from tests employing simulated earthquake excitations.

\subsection{Sinusoidal excitation response}

In every test the values of mass of the Block $\left(\mathrm{M}_{1}\right)$ as well as of mass of the S.D.O.F. $\left(\mathrm{M}_{2}\right)$ are stated; moreover, the values of the eigen-frequency of the S.D.O.F. together with that of frequency of the sinusoidal horizontal excitation are noted. Figures $9 \mathrm{a}$ to $9 \mathrm{~d}$ depict the
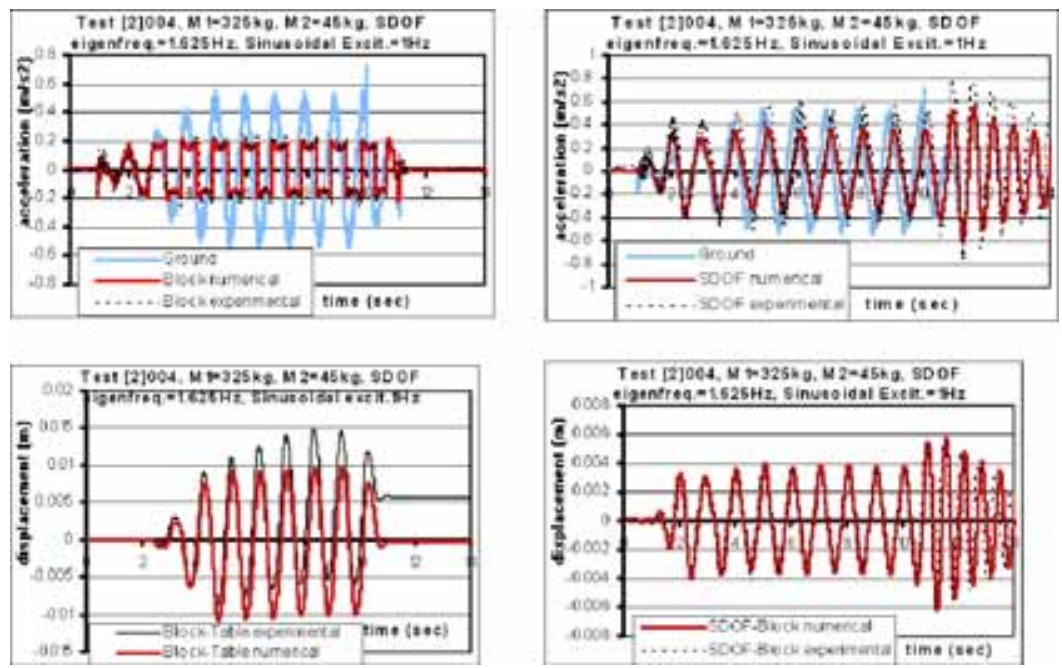

Figure 9: (a) Table (Ground) and Block (observed and predicted) acceleration response. (b) Table (Ground) and S.D.O.F. (observed and predicted) acceleration response. (c) Sliding displacement of the Block (predicted and observed). (d) Displacement of the S.D.O.F. relative to the Block (predicted and observed). 
numerical predictions and the experimental measurements for a test with sinusoidal excitation of $1 \mathrm{~Hz}$ when the eigen-frequency of the S.D.O.F. was $1.625 \mathrm{~Hz}\left(\mathrm{M}_{1}=325 \mathrm{~kg}\right.$ and $M_{2}=45 \mathrm{~kg}$ ). As can be seen in Fig. 9a and b, due to sliding of the rigid block, the acceleration response of either the Block (Fig. 9a) or the S.D.O.F. (Fig. 9b) are relatively small with no noticeable amplification when compared to the acceleration of excitation. As can be seen in these figures, the numerical prediction of either the Block acceleration response (Fig. 9a) or the S.D.O.F. acceleration response (Fig. 9b) are in reasonably good agreement with the corresponding experimental measurements. Similarly, good agreement can also be observed between the numerical predictions and experimental measurements of the Block sliding displacement response (Fig. 9c) or the displacement response of the S.D.O.F. relative to the Block (Fig. 9d). It must be underlined here that the acceleration of the excitation, as recorded by the acceleration sensor placed on the shaking table (Figs 8a and 8b), was directly used as input in the software described in Section 5.

Similar comparison between numerical predictions and measurements can be seen in Figs 10a to $10 \mathrm{~d}$. This time, due to the increase of the mass of the S.D.O.F. from $45 \mathrm{~kg}$ to $201 \mathrm{~kg}$, the value of the S.D.O.F. eigen-frequency is equal to $1.125 \mathrm{~Hz}$, which is quite close to the excitation frequency that remains the same as before $(1.0 \mathrm{~Hz})$. Good agreement can also be observed between the numerical predictions and experimental measurements of both the response of the Block and of the S.D.O.F. (Fig. 10a-d). If sliding was not allowed (fixed Block) for such an S.D.O.F. system with viscous damping ratio value of the order of $1 \%$, the S.D.O.F. acceleration response would be expected to have an amplification value of the order of 4.22 compared to the acceleration of the excitation. Due to sliding of the rigid Block (Fig. 10c), the acceleration response of either the Block (Fig. 10a) or of the S.D.O.F. (Fig. 10b) do not exhibit noticeable amplification when compared to the acceleration of the excitation,
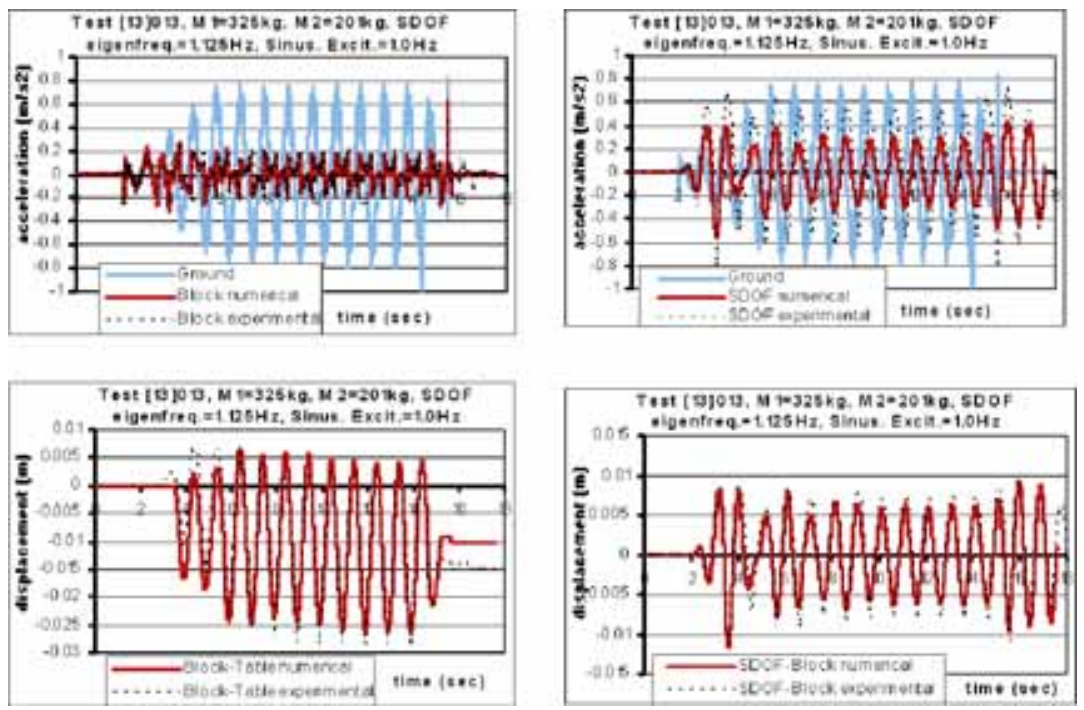

Figure 10: (a) Table (Ground) and Block (observed and predicted) acceleration response. (b) Table (Ground) and S.D.O.F. (observed and predicted) acceleration response. (c) Sliding displacement of the Block (predicted and observed). (d) Displacement of the S.D.O.F. relative to the Block (predicted and observed). 
despite the fact that the excitation frequency is quite close to the eigen-frequency of the S.D.O.F. The displacement of the S.D.O.F. relative to the Block attains maximum value of the order of $11 \mathrm{~mm}$ (Fig. 10d), which is larger than the corresponding value during the previous test $(6.5 \mathrm{~mm}$, Fig. 9d).

Figures 11a to $11 \mathrm{~d}$ depict the numerical predictions and the experimental observations for a test with sinusoidal excitation of $2.984 \mathrm{~Hz}$ when the eigen-frequency of the S.D.O.F. was $2.687 \mathrm{~Hz}\left(\mathrm{M}_{1}=325 \mathrm{~kg}\right.$ and $\left.\mathrm{M}_{2}=201 \mathrm{~kg}\right)$. Good agreement can be observed between the numerical predictions and experimental measurements of the response of the Block as well as the S.D.O.F. (Figs 11a-11d). If sliding was not allowed (fixed Block), for an S.D.O.F. system with viscous damping ratio value of the order of $1 \%$, the S.D.O.F. acceleration response would be expected to have an amplification value of the order of 4.27 compared to the acceleration of the excitation. Due to sliding of the rigid Block (Fig. 11c), the acceleration response of either the Block (Fig. 11a) or the S.D.O.F. (Fig. 11b) do not exhibit noticeable amplification when compared to the acceleration of the excitation, despite the fact that the excitation frequency is quite close to the eigen-frequency of the S.D.O.F. As also discussed in Section 2.4, it is interesting to note that the cumulative sliding displacements that appear as an offset from the initial zero displacement condition is successfully reproduced by the predicted response only in some cases (Fig. 10c) and not in other cases (Figs 9c and 11c). Possible explanations that are given for this are manufacturing tolerances, where the contact surface between the Block and the shaking table deviates from the ideal horizontal plane, thus resulting in somewhat larger maximum friction force in one direction than the maximum friction force in the opposite direction, as well as the integration scheme that is included in the computer software.
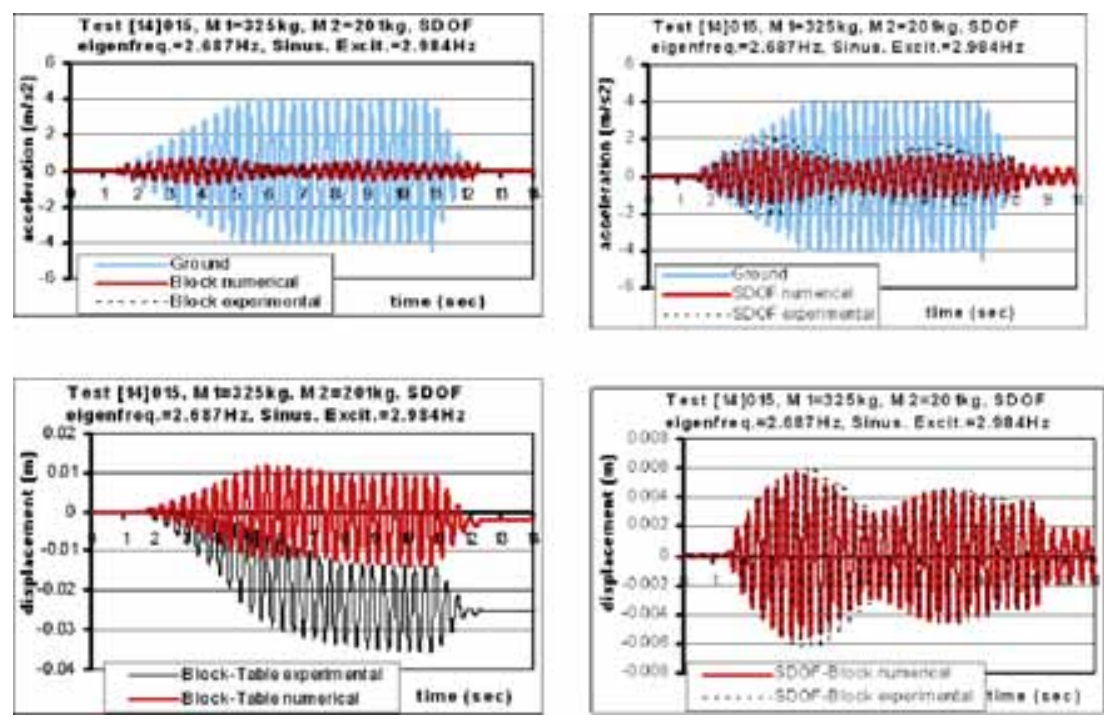

Figure 11: (a) Table (Ground) and Block (observed and predicted) acceleration response. (b) Table (Ground) and S.D.O.F. (observed and predicted) acceleration response. (c) Sliding displacement of the Block (predicted and observed). (d) Displacement of the S.D.O.F. relative to the Block (predicted and observed). 

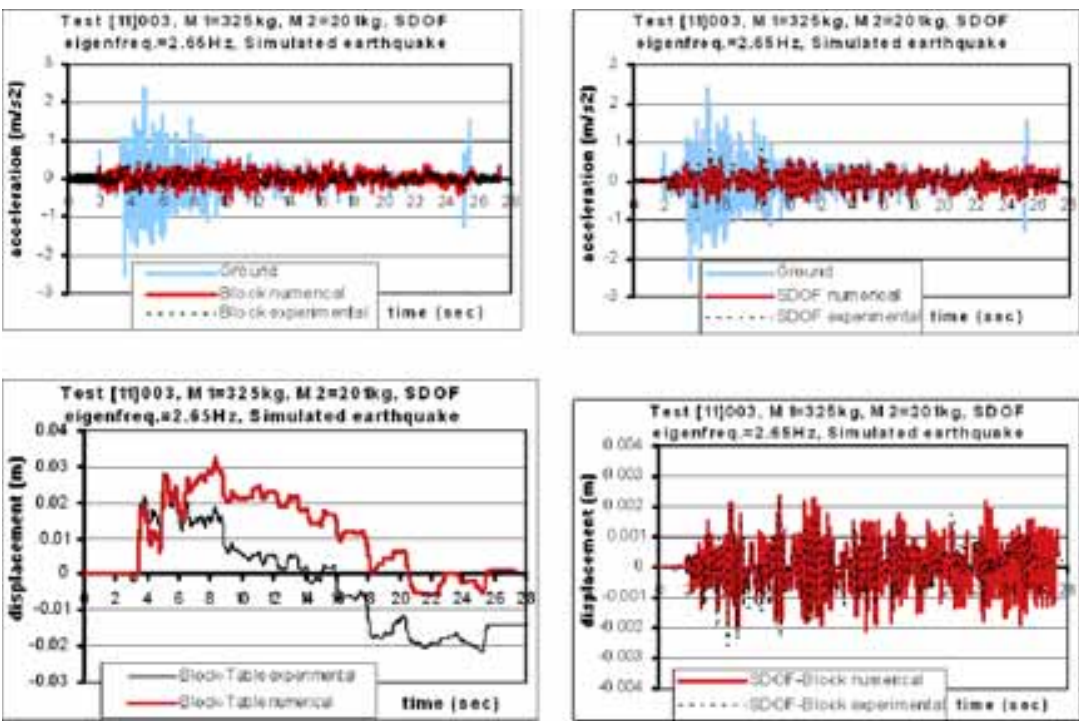

Figure 12: (a) Table (Ground) and Block (observed and predicted) acceleration response. (b) Table (Ground) and S.D.O.F. (observed and predicted) acceleration response. (c) Sliding displacement of the Block (predicted and observed). (d) Displacement of the S.D.O.F. relative to the Block (predicted and observed).

\subsection{Simulated horizontal earthquake excitations}

Figures $12 \mathrm{a}$ to $12 \mathrm{~d}$ depict the numerical predictions and the experimental observations for a test with simulated earthquake excitation, based on the prototype Kern County 1953 prototype earthquake horizontal acceleration recording.

The comparison between numerical predictions and experimental measurements in the case of simulated earthquake excitation is similar to the one commented upon in Section 6.1 for the sinusoidal excitation. Reasonably good agreement between measured and predicted response for both the Block and the S.D.O.F. can be seen in Figs 12a to 12d.

\section{CONCLUSIONS}

An investigation has been presented that studies the response of a 2-D.O.F. dynamic sliding system; that is an S.D.O.F. oscillator being fixed on top of a Block resting on a support that can slide along a horizontal axis. The response of this system is studied when subjected to unidirectional dynamic or earthquake excitations along this horizontal axis. This problem appears to be of interest in predicting the dynamic and earthquake response of superstructures supported on a large foundation block capable of horizontal sliding by means of seismic sliding isolators. Special mock-ups are tested in the present study at the shaking table of Aristotle University for this purpose utilizing horizontal simulated earthquake excitations based on prototype earthquake ground motion recordings. Moreover, numerical results were obtained by a computer software developed for this purpose. They are compared with the 
corresponding experimental measurements. The following conclusions can be drawn based on the presented material and the preceding discussion:

- The numerical predictions confirm all the behavioral observations recorded during the experimental sequence. Due to rigid block sliding, the acceleration response of the rigid block or the S.D.O.F., which is an oscillator with a relatively very low damping ratio value, does not exhibit noticeable amplification when compared to the acceleration of the excitation, despite the fact that the excitation frequency is quite close to the eigen-frequency of the S.D.O.F.

- The numerically predicted rigid block and S.D.O.F. acceleration response is in good agreement with the measured values for all examined cases for both the sinusoidal and the simulated earthquake horizontal excitation of the shaking table.

- The predicted rigid block sliding displacement response exhibited, in some cases, good agreement with the measured values. However, in other cases, significant deviation could be observed between the predicted and measured rigid block sliding displacement response values. The cumulative sliding rigid block displacement, that appears as an offset from the initial zero displacement condition, is successfully reproduced by the predicted response only in some cases.

- These deviations may be attributed to the actual sliding conditions due to manufacturing tolerances of the used mock-ups, whereby the contact surface between the rigid block and the shaking table deviates from the ideal horizontal plane. These actual sliding conditions result in somewhat larger maximum friction force in one direction than the maximum friction force in the opposite direction.

- The observed discrepancies between the measured and numerically predicted maximum sliding and cumulative sliding displacement response values may also be due to the integration scheme included in the computer software.

- These deviations between predicted and measured maximum and cumulative sliding displacement response are considerably reduced with the presence of a spring connecting the rigid block with the shaking table. Inverted pendulum sliding isolators, apart from sliding, possess such a spring balancing force property.

- It is demonstrated that the developed software can yield reasonably accurate predictions of the dynamic and earthquake response of the examined 2-D.O.F. sliding system. It can thus be utilized, in this simplified manner, for the preliminary design of the earthquake response of complex structural systems resting on sliding seismic isolators.

\section{REFERENCES}

[1] Aslam, M., Godden, W.C. \& Scalise, L.G., Sliding response of rigid bodies to earthquake motions, Rep. No. LBL-368, Lawrence Berkeley Laboratory, University of California, 1975.

[2] Westermo, B. \& Udwadia, F., Periodic response of sliding oscillator system to harmonic excitation. Earthquake Engineering and Structural Dynamics, 11, pp.135-146, 1983. doi: http://dx.doi.org/10.1002/eqe.4290110111

[3] Shenton, H.W. \& Jones, N.P., Base excitation of rigid bodies, I: Formulation. Engineering Mechanics, ASCE, 117(10), pp. 2286-2306, 1991. doi: http://dx.doi.org/ 10.1061/(ASCE)0733-9399(1991)117:10(2286)

[4] Virgin, L.N., Aldemir, U., Gavin, H. P., Nichols, J. M. \& Plaut, R. H., An investigation of a rocking-sliding block. ASME Design Engineering Technical Conference, 2001. 
[5] Demosthenous, M., Experimental and numerical study of the dynamic response of solid or sliced rigid bodies, Ph D Thesis, Dept. of Civil En., Aristotle University, Greece, 1994.

[6] Manos G.C., Demosthenous, M., Hatzigeorgiou, A. \& Manou-Evison, J., Study of the dynamic and earthquake behavior of models of ancient columns and colonnades with and without the inclusion of wires with energy dissipation characteristics, proc. 12th WCEE, 2000.

[7] Mostaghel, N. Hejazi, M. \& Tanbakuchi, J., Response of sliding structures to harmonic support motion. Earthquake Engineering and Structural Dynamics, 11, pp. 355-366, 1983. doi: http://dx.doi.org/10.1002/eqe.4290110305

[8] Mostaghel, N. \& Tanbakuchi, J., Response of sliding structures to earthquake support motion. Earthquake Engineering and Structural Dynamics, 11, pp. 729-748, 1983. doi: http://dx.doi.org/10.1002/eqe.4290110603

[9] Krishnamoorthy, A. \& Parikh, S., In-Plane response of a symmetric space frame with sliding supports. International Journal of Applied Science and Engineering, 3(1), pp. 1-11, 2005.

[10] Higashino M., Hamaguchi H., Minewaki S. \& Aizawa S., Basic characteristics and durability of low-friction sliding bearings for base isolation. Earthquake Engineering and Engineering Seismology, 4(1), pp. 95-105, 2003.

[11] Chopra, A. K., Dynamics of Structures: Theory and Applications to Earthquake Engineering, Prentice Hall: New Jersey, 1995.

[12] Manos, G.C., Koidis, G. \& Demosthenous, M., Investigation of the sliding response of a rigid body system subjected to uni-direction horizontal dynamic and earthquake excitations, proc. 3rd COMPDYN, Corfu: Greece, 2011.

[13] Choia B., and Tung C.C., Estimating sliding displacement of an unanchored body subjected to earthquake excitation. Earthquake Spectra, 18(4), pp. 601-613, 2002. doi: http://dx.doi.org/10.1193/1.1516750

[14] Wang Yen-Po, Chung Lap-Loi, \& Liao Wei-Hsin, Seismic response analysis of bridges isolated with friction pendulum bearings. Earthquake Engineering \& Structural Dynamics, 27(10), pp. 1069-1093, 1998. doi: http://dx.doi.org/10.1002/ (SICI) 1096-9845(199810)27:10<1069::AID-EQE770>3.0.CO;2-S

[15] Zayas, V., Low, S.S. \& Mahin, S.A., The FPS earthquake resisting system, experimental report, Report No. UCB+EERC-87/01, Berkeley, CA., 1987.

[16] Madden, G.J., Wongprasert, N. \& Symans, M. D., Analytical study of an adaptive sliding base isolation system for seismic protection of buildings, Proc. 14th ASCE Eng. Mechanics Conf. U.S.A., 2000. 